\title{
Super-LOTIS \\ A High-Sensitive Optical Counterpart Search Experiment
}

\author{
H. S. Park ${ }^{1}$, E. Ables ${ }^{1}$, D. L. Band ${ }^{5}$, S. D. Barthelmy ${ }^{3}$, R. M. \\ Bionta $^{1}$, P. S. Butterworth ${ }^{3}$, T. L. Cline ${ }^{3}$, D. H. Ferguson ${ }^{6}$, G. J. \\ Fishman $^{4}$, N. Gehrels ${ }^{3}$, D. Hartmann ${ }^{2}$, K. Hurley ${ }^{7}$, C. Kouveliotou ${ }^{4}$, \\ C. A. Meegan ${ }^{4}$, L. Ott ${ }^{1}$, E. Parker ${ }^{1}$, G. G. Williams ${ }^{2}$ \\ ${ }^{1}$ Lawrence Livermore National Laboratory, Livermore, CA 94550 \\ ${ }^{2}$ Dept. of Physics and Astronomy, Clemson University, Clemson, SC 29634-1911 \\ ${ }^{3}$ NASA/Goddard Space Flight Center, Greenbelt, MD 20771 \\ ${ }^{4}$ NASA/Marshall Space Flight Center, Huntsville, AL 35812 \\ ${ }^{5}$ CASS 0424, University of California, San Diego, La Jolla, CA 92093 \\ ${ }^{6}$ Dept. of Physics, California State University at Hayward, Hayward, CA 94542 \\ ${ }^{7}$ Space Sciences Laboratory, University of California, Berkeley, CA 94720-7450
}

\begin{abstract}
We are constructing a 0.6 meter telescope system to search for early time gamma-ray burst(GRB) optical counterparts. Super-LOTIS (Super-Livermore Optical Transient Imaging System) is an automated telescope system that has a $0.8 \times 0.8^{\circ}$ field-of-view, is sensitive to $\mathrm{Mv} \sim 19$ and responds to a burst trigger within 5 min. This telescope will record images of the gamma-ray burst coordinates that is given by the GCN (GRB Coordinate Network). A measurement of GRB light curves at early times will greatly enhance our understanding of GRB physics.
\end{abstract}

\section{INTRODUCTION}

The origin and nature of gamma-ray bursts (GRBs) remains an important unresolved problem in astrophysics. GRBs are brief bursts $(<100 \mathrm{sec}$ duration) of high-energy radiation that appear at random in the sky. Much of the difficulty in studying gamma-ray bursts results from the poor directional precision $\left(1 \sim 10^{\circ} 1-\sigma\right.$ statistical error) available from current gamma-ray detection experiments and their short duration $(1 \sim 100 \mathrm{sec})$. Even though recent fading x-ray, optical and radio counterpart observations by the Italian-Dutch satellite (BeppoSAX) [1], [2], [3] provided information on their distance scale [4], these observations were made many hours later than the GRB. These afterglows may be due to different process from the GRB production mechanism. An observation of optical activity simultaneous 
to the GRB may provide clues to understanding this process.

In an attempt to search for simultaneous optical counterparts of GRBs, we initially utilized an existing wide-field-of-view telescope at Lawrence Livermore National Laboratory (LLNL) to rapidly image GRB coordinates distributed by the BATSE real-time coordinate distribution network [5]. This first experiment, the Gamma Ray Optical Counterpart Search Experiment (GROCSE), did not find optical optical counterparts at the $\mathrm{Mv} \sim 7.5$ sensitivity level [6].

Subsequently, we constructed the Livermore Optical Transient Imaging System (LOTIS) which has a $17.4 \times 17.4^{\circ}$ field of view to image the entire error circle of the rapid GCN notice (BATSE-Original trigger coordinates arrive in 5 sec but have $\sim 15^{\circ}$ errors). LOTIS has been operating since Oct. 1996 and we have not yet observed any simultaneous optical activity at $\mathrm{Mv} \sim 11$ level [7], [8].

In order to provide better GRB coordinates to search for counterparts, the GCN has installed new triggers called LOCBURST and RXTE which utilize the best analysis performed by the BATSE team (involving an operator's interaction) and the RXTE satellite which uses the hard x-ray afterglow to calculate a better position. The LOCBURST trigger error is $0.2 \sim 2^{\circ}$; and the RXTE trigger error is $6 \sim 40$ arcmin depending on the statistics. Although delayed $(15 \sim 35 \mathrm{~min}$. for LOCBURST; $3 \sim 5 \mathrm{hr}$. for RXTE triggers), the smaller error box enables a more conventional, deeper telescope (larger aperture but smaller field of view than LOTIS) to follow up on the GRBs. Since the bursts are random, this telescope will need to be dedicated and automated to always be ready for new triggers.

In an attempt to make such observations, we are constructing a large aperture telescope system dedicated to this search.

\section{SUPER-LOTIS}

The telescope is a Boller and Chivens 0.6 meter reflective telescope with $\mathrm{f} / 3.5$. Figure 1 shows the telescope. It has superb optical quality and mechanical structure; however, it is not equipped with computer controllable drives nor an electrooptical imaging sensor. We are converting this telescope to Super-LOTIS by refurbishing the motor drive, installing a CCD camera, and placing it at a remote site for dedicated observation. As for the sensor, we are installing a LOTIS CCD camera which utilizes a Loral 442A 2048 x 2048 CCD (15 x $15 \mu \mathrm{m}$ pixels) with LLNL built readout electronics. The CCD is cooled by thermo electric cooling (to $-30^{\circ} \mathrm{C}$ ) which minimizes dark current and readout noise.

The Super-LOTIS will have $0.84 \times 0.84^{\circ}$ field-of-view (1.5 arcsec/pixel) which is sufficient for BATSE/RXTE trigger types distributed by the GCN. We plan to dither around the GCN "Original" trigger coordinates which has only a 5 sec delay, but a large $15^{\circ}$ error box. When we receive refined positions, i.e. LOCBURST, or RXTE triggers, we will scan the region and stay at that location the rest of the night. Our scanning strategy and automation will allow us to record GRB optical activity as early as a few minutes. 
The basic on-line software has already been written and has been operating on LOTIS. We will need very minimal modification to the existing software for the entire data acquisition control.

We have estimated the sensitivity of the Super-LOTIS system. The calculation includes the measured camera dark current at $-30^{\circ} \mathrm{C}$, readout noise, typical sky background at an observing site and shot noise. Figure 2 shows the resulting signal to noise ratio with 10 and $60 \mathrm{sec}$ integration time vs. the visual magnitude. The calculation indicates that the Super-LOTIS will see Mv $\sim 19$ stars at a signal to noise ratio of 10 with $10 \mathrm{sec}$ and $\mathrm{Mv} \sim 21$ with $60 \mathrm{sec}$ integration times.

Utilizing our successful experience in construction, operation, data handling, and data analysis of the GROCSE and LOTIS systems we expect Super-LOTIS to be constructed and operational within a year. Detection of optical emission at early times (or placing stringent constraints) would provide a crucial link between the multiwavelengths properties of the burst and its afterglow. Burst and afterglow emission are likely to probe different aspects of the GRB model (e.g. internal

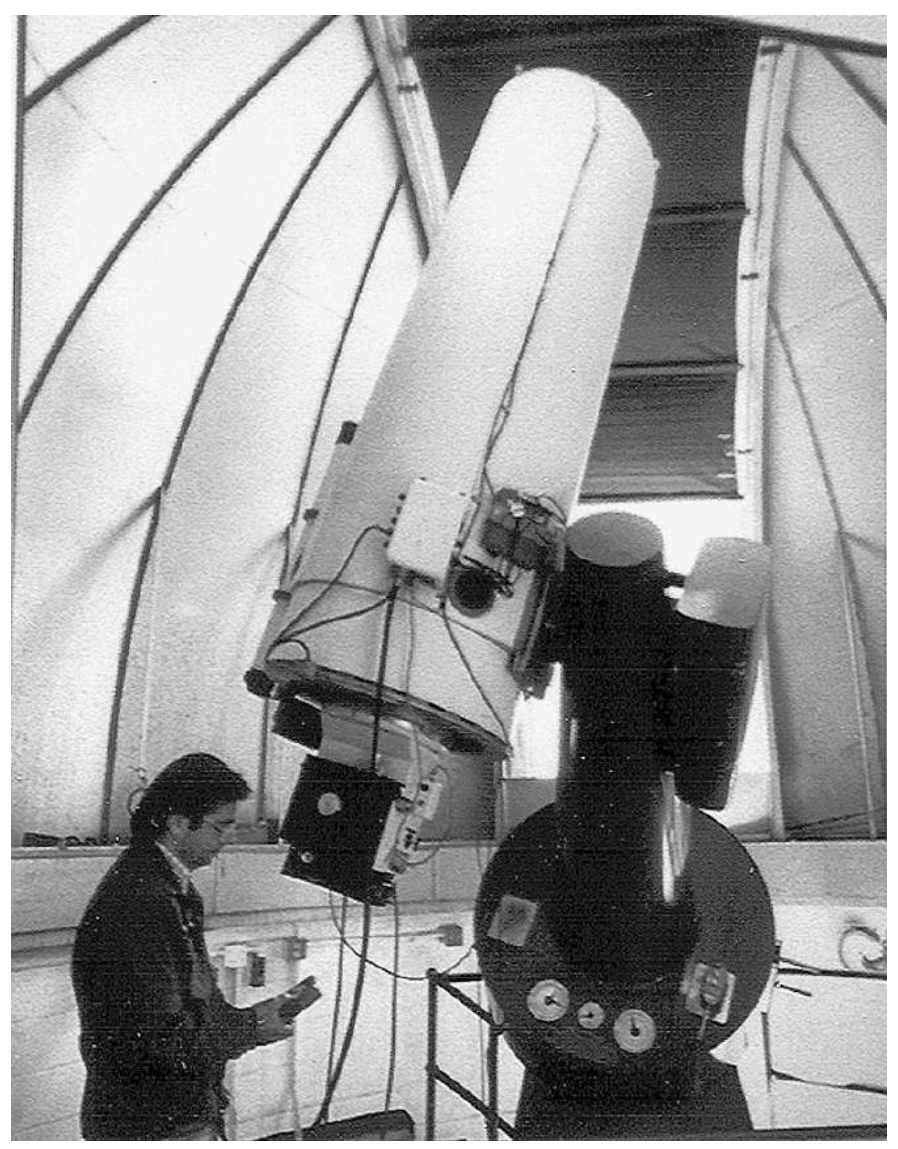

FIGURE 1. Super-LOTIS Boller and Chivens 0.6 meter reflective telescope. After refurbishing by adding computer controlled motors and installing a CCD camera, this automated system will be dedicated to the GRB optical counterpart search. 
vs. external shocks.) So far only two afterglows have been detected and no optical detection simultaneous with or shortly after the gamma-ray burst has been made. The 90 minute delayed emission of high energy photons from GRB940217 [9] suggests that similar emissions in the optical wavelength could accompany some bursts. Super-LOTIS would detect such a new spectral component of the bursts to a magnitude level of $>\mathrm{Mv} \sim 19$. While upper limits will be useful for constraining the models, Super-LOTIS will establish the GRB light curves at early times which will provide a crucial step toward understanding GRB phenomenon.

\section{REFERENCES}

1. Costa, E., et al., IAU Circ., 6572 (1997).

2. Costa, E., et al., IAU Circ., 6649 (1997).

3. Heise, J., et al., IAU Circ., 6654 (1997).

4. Metzger, M., et al., IAU Circ., 6655 (1997).

5. Barthelmy, A., et al., These Proceedings (1998).

6. Park, H., et al., AstroPhys. Journal, 490 (1997).

7. Park, H., et al., AstroPhys. Journal Letters, 490, L21-L24 (1997).

8. Williams, G., These Proceedings (1998).

9. Hurley, K., et al., Nature., 372, 652 (1994).

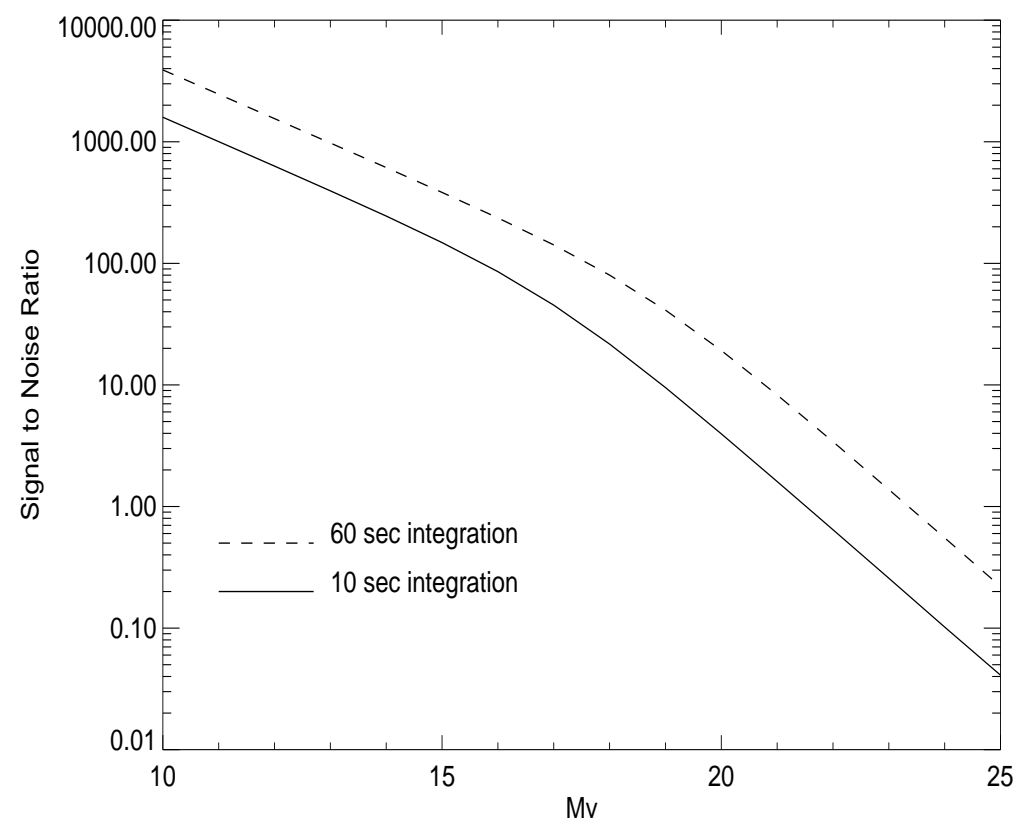

FIGURE 2. Super-LOTIS sensitivity: Predicted signal to noise ratio vs. visual magnitude. Super-LOTIS will be able to detect $\mathrm{Mv} \sim 19$ objects with $10 \mathrm{sec}$ and $\mathrm{Mv} \sim 21$ with $60 \mathrm{sec}$ integration times. 